\title{
Regulation of the dorsal morphogen by the Toll and torso signaling pathways: a receptor tyrosine kinase selectively masks transcriptional repression
}

\author{
Jannette Rusch and Michael Levine \\ Department of Biology, Center for Molecular Genetics, University of California-San Diego, \\ La Jolla, California 92093-0322 USA
}

\begin{abstract}
The dorsal $(d l)$ nuclear gradient initiates the differentiation of the mesoderm, neuroectoderm, and dorsal ectoderm by activating and repressing gene expression in the early Drosophila embryo. This gradient is organized by a Toll signaling pathway that shares many common features with the mammalian IL-1 cytokine pathway. Here we present evidence that a second signaling pathway, controlled by the torso (tor) receptor tyrosine kinase, also modulates $d l$ activity. Evidence is presented that the tor pathway selectively masks the ability of $\boldsymbol{d l}$ to repress gene expression but has only a slight effect on activation. Intracellular kinases that are thought to function downstream of tor, such as D-raf and the rolled MAP kinase, mediate this selective block in repression. Normally, the Toll and tor pathways are both active only at the embryonic poles, and consequently, target genes (zen and $d p p$ ) that are repressed in middle body regions are expressed at these sites. Constitutive activation of the tor pathway causes severe embryonic defects, including disruptions in gastrulation and mesoderm differentiation, as a result of misregulation of $d l$ target genes. These results suggest that RTK signaling pathways can control gene expression by antirepression, and that multiple pathways can fine-tune the activities of a single transcription factor.
\end{abstract}

[Key Words: dorsal morphogen; Toll; torso; receptor tyrosine kinase; transcriptional repression]

Received March 16, 1994; revised version accepted April 29, 1994.

The dorsal (dl) morphogen initiates the differentiation of the embryonic mesoderm, neuroectoderm, and dorsal ectoderm (for review, see Govind and Steward 1991; Ip and Levine 1992; St Johnston and Nüsslein-Volhard 1992). dl is a member of the REL family of transcription factors (Steward 1987), which are all regulated at the level of nuclear transport (Liou and Baltimore 1993). In the case of $d l$, selective nuclear transport results in the formation of a broad protein gradient, with peak levels present in ventral regions of early embryos and progressively lower levels in lateral and dorsal regions (Roth et al. 1989; Rushlow et al. 1989; Steward 1989).

Peak levels of $d l$ activate the expression of regulatory genes such as twist (twi) and snail (sna) in ventral regions; these are required for initiating the differentiation of the mesoderm (Boulay et al. 1987; Thisse et al. 1987). Low levels of $d l$ trigger the expression of neuroectodermal regulatory genes in lateral regions, which define the neurogenic territory (Ip et al. 1992a). dl may be unique among the maternal gradients of transcription factors by functioning as both a transcriptional activator and repressor (Jiang et al. 1992; Pan and Courey 1992). dl not only activates mesodermal and neuroectodermal target genes in a concentration-dependent manner but also represses the expression of certain genes, such as zerknüllt (zen) and decapentaplegic (dpp), thereby restricting them to the dorsal ectoderm (Doyle et al. 1989; Ip et al. 1991; Huang et al. 1993; Jiang et al. 1993; Kirov et al. 1993), where they are important for the differentiation of the amnioserosa and dorsal epidermis, respectively (Wakimoto et al. 1984; St. Johnston and Gelbart 1987). In $\mathrm{dl}^{-}$ embryos there is a failure to activate mesoderm and neuroectoderm determinants, so derivatives of these embryonic tissues do not form (Simpson 1983). Moreover, zen and $d p p$ are not restricted to dorsal regions but are instead expressed throughout $\mathrm{dl}^{-}$mutants /see Fig. 1C; Rushlow et al. 1987). This derepression causes all cells of dl mutants to differentiate into derivatives of the dorsal ectoderm.

The formation of the $d l$ gradient depends on a cell signaling pathway, which includes a transmembrane receptor, Toll, as well as several intracellular components, including a putative kinase, pelle (for review, see Govind and Steward 1991; Wasserman 1993). Several lines of evidence suggest that a cascade of extracellular serine proteases locally activate the spätzle ligand in ventral and polar regions of the perivitteline fluid that surrounds the egg and early embryo (Morisato and Anderson 1994). Activated spätzle locally interacts with Toll receptor in ventral regions and the poles. This leads to the dissoci- 
Figure 1. Regulation of zen by $d l$ and tor. All embryos are at the 14th nuclear cleavage cycle and are oriented with anterior to the left and dorsal up. Expression patterns were visualized by in situ hybridization using digoxygenin-UTP-labeled RNA probes. $(A)$ zen expression pattern in a wild-type embryo. Staining covers the dorsal half of the embryo and includes both poles (arrowheads). (B) zen expression in an embryo derived from a female homozygous for the strong tor $^{P M 51}$ mutation. Staining is lost from the poles (arrowheads) but is normal in middle body regions. $(C)$ zen expression in an embryo derived from a female homozygous for the $d l^{I 5}$ mutation. Staining is completely derepressed in ventral regions and the poles. Only the pole cells are unstained. $(D)$ zen expression in an embryo derived from a $d l^{15}$, tor ${ }^{P M 51}$ double mutant. The staining pattern is identical to that observed in $C$.
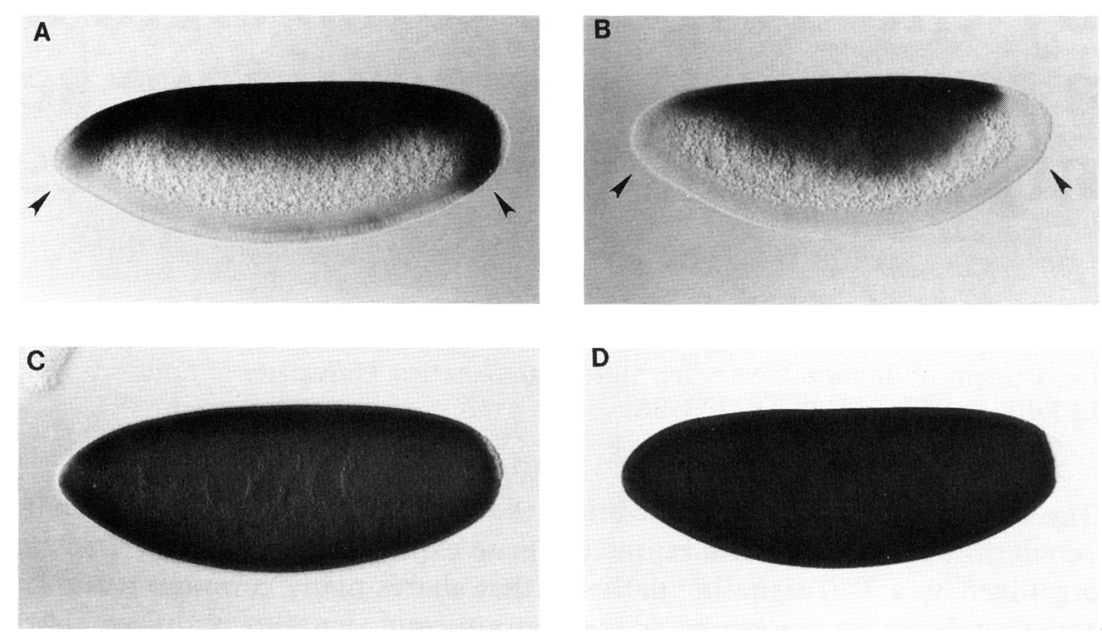

D

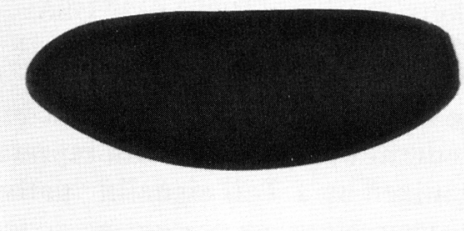

ation of an inactive cytoplasmic complex containing $d l$ and its inhibitor, cactus (cact), so that $d l$ is released to the nucleus where it functions as a transcriptional activator and repressor (Geisler et al. 1992; Kidd 1992).

There is considerable information regarding the way in which $d l$ regulates gene expression. Target promoters containing low-affinity $d l$-binding sites are activated only in ventral regions, the presumptive mesoderm, where there are peak levels of $d l$ protein (Jiang et al. 1991; Pan et al. 1991; Thisse et al. 1991; Ip et al. 1992b; Jiang and Levine 1993). In contrast, promoters containing high-affinity $d l$-binding sites and $\mathrm{E}$ boxes (recognition sequences for helix-loop-helix proteins) are activated in both ventral (presumptive mesoderm) and lateral (presumptive neuroectoderm) regions (Ip et al. 1992a; Jiang and Levine 1993). Restricted expression in the neuroectoderm depends on the sna repressor, which is localized to the mesoderm (Alberga et al. 1991; Kosman et al. 1991; Leptin 1991; Ip et al. 1992b).

$d l$ is intrinsically a weak transcriptional activator in the early embryo. The efficient activation of target promoters such as twi and sna depends on multiplicative interactions among numerous $\mathrm{dl}$-binding sites (Jiang et al. 1991; Pan et al. 1991; Thisse et al. 1991; Ip et al. 1992b). Moreover, dl-HLH interactions also augment the levels of target gene expression (Kosman et al. 1991; Ip et al. 1992b; González-Crespo and Levine 1993; Jiang and Levine 1993). Recent studies suggest that the $d l$ activator is converted into a potent repressor when it interacts with neighboring "corepressors" bound to the $z e n$ and $d p p$ promoters (Huang et al. 1993; Jiang et al. 1993; Kirov et al. 1993). For example, a minimal silencer element has been identified in distal regions of the zen promoter that can repress the ventral expression of heterologous promoters (Doyle et al. 1989; Ip et al. 1991; Jiang et al. 1993; Kirov et al. 1993). It contains several $d l$-binding sites as well as closely linked binding sites for one or more corepressors. These corepressors have not yet been characterized; however, there is evidence that they interact with $d l$ and foster cooperative binding to DNA (Jiang et al. 1992). Mutations in the corepressor sites convert the zen silencer element into a weak enhancer (Jiang et al. 1993; Kirov et al. 1993). Because the dl-binding sites are intact, they can mediate weak activation in response to high levels of $d l$ in ventral regions of early embryos. Only when both the $d l$ sites and the nearby corepressor sites are intact can $d l$ function as a repressor.

Although $d l$ represses zen and $d p p$ in ventral and lateral regions of the embryo, both genes are expressed at high levels at the poles (Doyle et al. 1986; St Johnston and Gelbart 1987). However, there are equally high levels of $d l$ in the nuclei of ventral and polar regions, and both sites of expression are lost in Toll- mutants (Roth et al. 1989; Steward, 1989|. Nonetheless, $d l$ fails to repress zen at the poles. Previous studies suggest that the torso (tor) receptor tyrosine kinase (RTK) plays a role in this process. zen normally is expressed in dorsal regions of tor mutants but is lost at the poles (Rushlow et al. 1987). This observation suggests that tor might regulate a transcriptional activator at the poles that overrides $d l$ mediated repression. Alternatively, it is possible that the tor signaling pathway somehow masks the ability of $d l$ to function as a repressor.

Here, we present evidence favoring the latter model, whereby tor functions as an antirepressor that blocks dl-mediated repression. Constitutive activation of the tor RTK causes zen to be completely derepressed in ventral regions of early embryos. However, target genes that are normally activated by $d l$ are only slightly affected, suggesting that $t$ tor selectively blocks the ability of $d l$ to function as a repressor but has little or no effect on $d l$ mediated activation. Intracellular kinases that are thought to function downstream of tor (for review, see 
Perrimon 1993) appear to mediate this selective block in repression. In particular, the removal of maternal $D$-raf products results in a loss of zen expression at the poles, indentical to that observed in tor mutants. Similarly, a hyperactivated form of the Drosophila MAP kinase homolog, rolled (Brunner et al. 1994; Biggs et al. 1994), attenuates $d l$-mediated repression. These results suggest that two independently acting signaling pathways, Toll and tor, fine-tune the activities of the $d l$ morphogen. In middle body regions $d l$ functions as both an activator and repressor because it is only regulated by Toll, but at the poles $d l$ functions only as an activator because of the combined action of both the Toll and tor pathways.

\section{Results}

In situ hybridization was used to monitor the effects of the tor signaling pathway on $d l$ activity. Embryos were collected from mutant females carrying various maternal-effect mutations and hybridized with digoxygeninlabeled RNA probes (Tautz and Pfeifle 1989; Jiang et al. 1991). This allowed the visualization of a number of different expression patterns, including endogenous genes that normally are activated or repressed by $d l$, as well as various defined synthetic promoters (see Jiang and Levine 1993).

\section{tor exerts an antirepression effect on $\mathrm{dl}$}

$z e n$ is an early target gene that is directly repressed by $d l$ (Jiang et al. 1992). Both high levels of $d l$ in ventral regions and low levels in lateral regions repress zen, so that its expression is restricted to the dorsal half of the embryo (Fig. 1A). In addition, the zen pattern extends into both poles, despite the high levels of nuclearly localized dl protein present at these sites (Roth et al. 1989; Rushlow et al. 1989; Steward 1989). In embryos derived from $\mathrm{dl}^{-}$females, the early zen pattern is derepressed throughout the embryo and includes all somatic cells (Fig. 1C). As shown previously, zen expression is lost from the poles in embryos derived from tor ${ }^{-}$females, although there is no effect on the normal dorsoventral limits of the pattern in middle body regions (Fig. 1B; Rushlow et al. 1987). This loss in polar expression could reflect a failure to induce a transcriptional activator or might result from an "antirepression" effect, whereby tor masks the ability of $d l$ to function as a transcriptional repressor.

In an effort to distinguish between these possibilities, we examined zen expression in embryos derived from $\mathrm{dl}^{-}$, tor $^{-}$double mutants (Fig. 1D). Staining is detected throughout the embryo, similar to the pattern observed in $\mathrm{dl}^{-}$mutants (Fig. 1, cf. D with C). The simplest interpretation of this result is that tor $^{+}$gene activity normally blocks $d l$ repression at the poles, but it is not required for polar expression in the absence of the $d l$ repressor. A similar result was obtained for the early pattern of $d p p$ expression, which is another target gene that is directly repressed by $d l$ (Casanova 1991).

\section{Ectopic activation of tor blocks $\mathrm{dl}$ repression throughout the embryo}

There are several dominant tor mutations that cause constitutive, ligand-independent activation of tor RTK activity (Casanova and Struhl 1989; Sprenger et al. 1989). Normally, tor is ubiquitously expressed on the cell surface of early embryos but is activated only at the poles by a localized ligand encoded by the torso-like (tsl) gene (Savant-Bhonsale and Montell 1993; Martin et al. 1994). Localized activation of the tor RTK results in the restricted expression of the gap gene tailless (tIl) at the poles (Fig. 2C). The tor ${ }^{4021}$ mutation causes the most severe constitutive activation of tor RTK activity among the known dominant tor alleles (Klingler et al. 1988; Casanova and Struhl 1989). Embryos derived from $\operatorname{tor}^{4021} /+$ females display a strongly abnormal $t 11$ expression pattern, such that staining is nearly ubiquitous and spans almost the entire length of the embryo (Fig. 2D). This ectopic expression of $t l l$ causes severe disruptions in the embryonic segmentation pattern (Steingrimsson

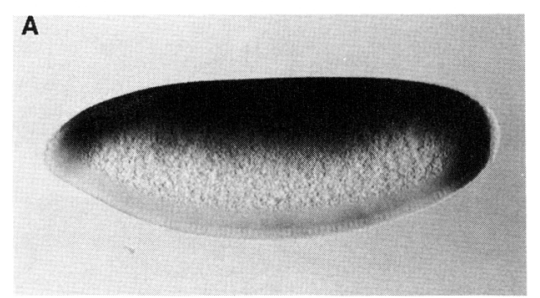

B
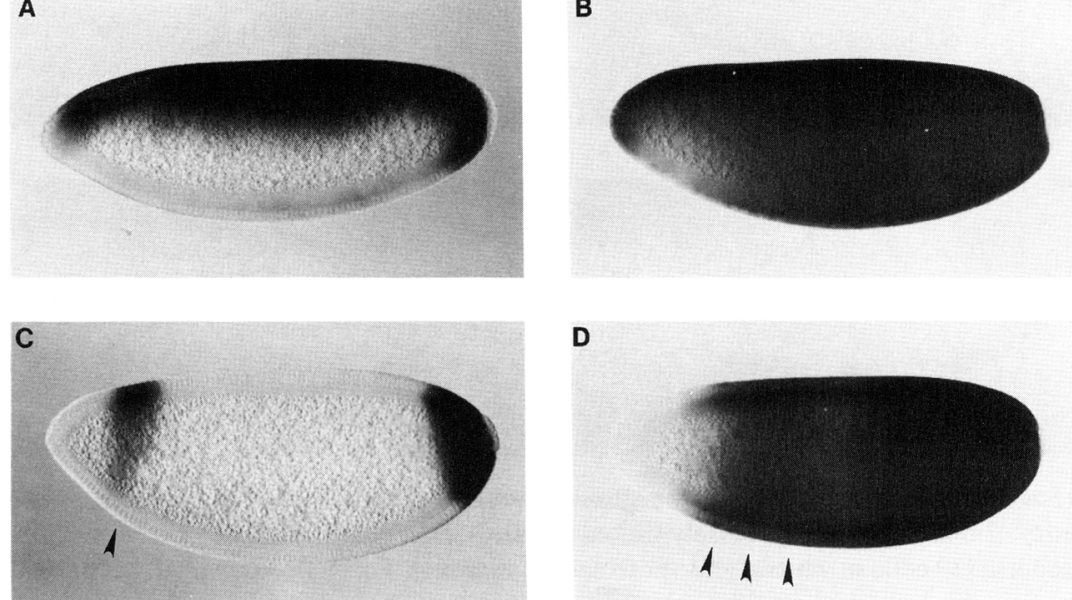

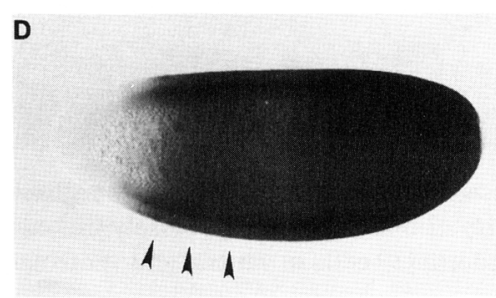

Figure 2. Constitutive activation of tor disrupts zen and tll expression. Embryos are staged and presented as in Fig. 1. $|A|$ Wildtype pattern of zen expression. $(B)$ zen expression pattern in an embryo derived from a tor $^{4021} /+$ female. Staining is strongly derepressed in ventral regions, similar to that observed in $\mathrm{dl}^{-}$mutants (cf. with Fig. $1 C$ ). (C) tll expression pattern in a wild-type embryo. Staining is restricted to the anterior and posterior poles, although the anterior pattern is repressed from ventral regions (arrowhead). (D) tll expression pattern in an embryo derived from a tor ${ }^{4021} /+$ female. Staining nearly encompasses the entire length of the embryo, and there is a derepression of the anterior pattern so that it now includes ventral regions (arrowheads; cf. with $C$ ). 
et al. 1991). The absence of expression in the anteriormost regions might reflect a slight expansion of the normal mechanisms that establish the anterior limit of the wild-type pattern (Fig. 2D; cf. D with C), including the attenuation of the bicoid ( $b c d)$ activator by the tor pathway (Ronchi et al. 1993).

Constitutive activation of tor also results in a dramatic derepression of the zen pattern in early embryos (Fig. 2B; cf. B with A). In some embryos, staining extends almost uniformly along the entire length of the embryo while in others (such as the one shown in Fig. 2B), there is a slight repression of the pattern in anterior, ventral regions. This site of residual repression activity corresponds roughly to the location of the ventral limit of the normal anterior $t 11$ stripe (see Fig. $2 \mathrm{C}$ ). The derepression of the zen pattern observed in tor ${ }^{4021}$ mutants is not the result of a block in $d l$ nuclear transport, because these mutants contain normal levels of nuclearly localized $d l$ repressor in ventral and ventrolateral regions (data not shown). This observation suggests that tor activity can mask the ability of $d l$ to function as a transcriptional repressor whenever the tor and Toll pathways coincide. In wild-type embryos, tor activity is restricted to the poles and normally blocks $d l$-mediated repression only in these regions. However, in embryos where tor is constitutively activated, $d l$ repression is blocked both at the poles and in middle body regions.

Previous studies suggest that $d l$ either directly or indirectly represses the anterior domain of the tll pattern in ventral regions (arrowhead in Fig. 2C). In $\mathrm{dl}^{-}$embryos this anterior pattern expands so that expression is uniformly intense in both dorsal and ventral regions ( $\mathrm{Pi}$ gnoni et al. 1992). Constitutive activation of tor results in a similar ventral derepression of the $t 11$ pattern (arrowheads in Fig. 2D). This result suggests that genetic interactions between $d l$ and tor lead to a general block in the ability of $d l$ to function as a transcriptional repressor. Consistent with this view is the finding that repression of the $d p p$ pattern is also impaired in tor gain-of-function mutants (data not shown). Another implication of this result is that $d l$ might directly repress $t 11$ expression. In principle, it is possible that ventral repression of the anterior tll pattern (arrowhead in Fig. 2C) could be mediated indirectly by a $d l$ target gene such as twi or sna. However, as shown below, constitutive activation of tor does not significantly disrupt the initiation of these genes.

\section{tor exerts only a slight effect on dl activation}

The twi and sna expression patterns were examined to determine whether dominant tor mutations influence the ability of $d l$ to function as a transcriptional activator (Fig. 3). Embryos were collected from tor $^{4021} /+$ females and stained to reveal the patterns of endogenous twi and sna RNAs. The initiation of both patterns is essentially normal (Fig. 3A,C), although there is a severe premature loss in sna expression by the onset of gastrulation. This is associated with defects in ventral furrow formation and mesoderm invagination (data not shown). sna is normally repressed from the posterior pole of wild-type embryos (Alberga et al. 1991; Kosman et al. 1991; Leptin 1991), and it seems likely that the expanded limits of sna repression observed in tor gain-of-function mutants result from expanded expression of one or more tor target genes, such as huckebein $(h k b)$ or $t 11$ (Brönner and Jäckle 1991; see Discussion).

Further evidence that tor has little or no effect on the ability of $d l$ to work as an activator was obtained by analyzing the expression of synthetic promoters. Previous studies have shown that a truncated sna promoter containing the first $1.6 \mathrm{~kb}$ of sna $5^{\prime}$-flanking sequence is
Figure 3. Constitutive activation of tor causes only mild defects in the initiation of target genes. All embryos were derived from tor $4021 /+$ females. $(A)$ twi expression pattern. Staining extends along the length of the ventral surface and encompasses the poles. This pattern is quite similar to that observed in wild-type embryos (not shown). (B) Expression of a lac $Z$ fusion gene containing the first $1.6 \mathrm{~kb}$ of the sna promoter. Staining encompasses ventral regions but does not extend to the poles. This pattern is quite similar to that observed in wild-type embryos (Ip et al. 1992b), although there is about a two-fold reduction in the levels of staining. $(C)$ sna expression pattern. Staining includes ventral regions and the poles. This early pattern is quite similar to that observed in wild-type embryos (not shown), although there is a progressive weakening in expression particularly in posterior regions (arrowheads). (D) Expression pattern of the synthetic PE-Et promoter, which contains low-affinity $d l$-binding sites and closely linked twi sites (Et E boxes). Staining extends to the poles and encompasses the entire presumptive mesoderm ( 20 cells in ventral and ventrolateral regions). This pattern is nearly identical to that observed in wild-type embryos.
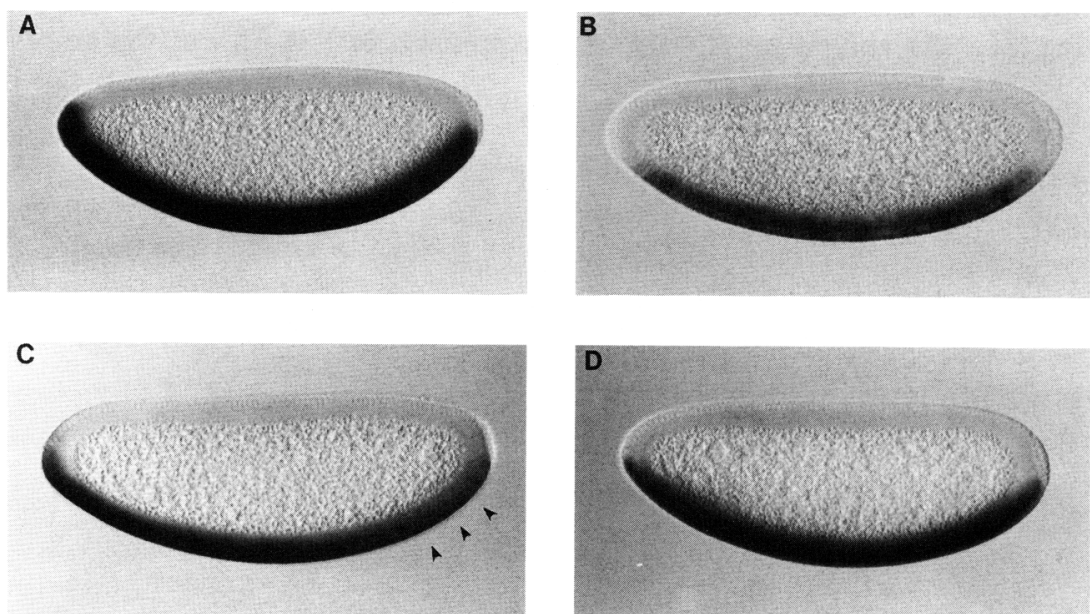
activated only by peak levels of $d l$ protein present in the ventral-most 12 cells of early embryos (Ip et al. 1992b). A lac $Z$ fusion gene containing this promoter sequence can be thought of as a "sensitized" element because just a two-fold reduction in the levels of $d l$ (embryos derived from $\mathrm{dl}^{-} /+$mothers) causes nearly a complete loss in expression. Consequently, it provides a sensitive assay for investigating the effects of tor on $\mathrm{dl}$-mediated activation. The sna-lacZ fusion gene was crossed into an embryo derived from $t_{0}{ }^{4021} /$ + females, and its expression was monitored by in situ hybridization using a digoxygenin-labeled $l a c Z$ antisense RNA probe. An essentially normal pattern is observed, with expression spanning the entire ventral surface of the embryo (Fig. 3B). However, there is a mild reduction in the levels of expression and a slight narrowing of the pattern ( 10 cells rather than 12). Nonetheless, this result suggests that the constitutive activation of tor RTK activity causes less than a two-fold reduction in the effective levels of $d l$ activator.

The $d l$ protein is distributed in a steep concentration gradient so that there are substantially lower levels of protein in ventrolateral versus ventral regions (Roth et al. 1989; Rushlow et al. 1989; Steward 1989). To determine whether tor might disrupt the ability of intermediate levels of $d l$ to activate gene expression, we analyzed the expression of a synthetic twi promoter fragment, PE-Et. This 440-bp regulatory sequence directs expression in the entire presumptive mesoderm (spanning $\sim 18$ cells in width), including both ventral and ventrolateral regions of early embryos (Jiang and Levine 1993). The normal PE sequence directs expression in the ventral-most $10-12$ cells, similar to the pattern generated by the 1.6-kb sna-lacZ fusion gene (Jiang et al. 1991; Ip et al. 1992b). A modified PE containing two recognition sequences for the $t w i$ protein $\left({ }^{\prime \prime} E t^{\prime \prime}\right)$ directs an expanded pattern that includes ventrolateral regions where there are intermediate levels of $d l$ (Jiang and Levine 1993). A PE-Et-lacZ fusion gene shows a normal pattern of expression in tor ${ }^{4021}$ mutants (Fig. 3D), with expanded dorsoventral limits as seen in wild-type embryos. These results suggest that tor-dl interactions do not impair dl-mediated activation in either ventral or ventrolateral regions.

Uncoupling of $d l$-mediated activation versus repression is observed most dramatically with fusion genes containing the zen silencer element. Previous studies have shown that $d l$ represses zen in ventral regions by interacting with a 180-bp ventral repression element (VRE) located $\sim 1.4 \mathrm{~kb}$ upstream of the transcription start site (Doyle et al. 1989; Ip et al. 1991; Jiang et al. 1992, 1993; Kirov et al. 1993). This element contains three high-affinity $d l$-binding sites, as well as closely linked binding sites for one or more corepressors. The $d l$ protein is intrinsically a weak transcriptional activator; however, interactions between $d l$ and neighboring corepressors in the zen VRE convert $d l$ into a repressor that silences the ventral expression of heterologous promoters. For example, ventral expression of even-skipped (eve) stripe 2 is silenced when the zen VRE is placed upstream of the 480-bp eve minimal stripe 2 enhancer ("MSE"; Fig. 4A). Mutations in the corepressor binding sites abolish the ability of the zen VRE to function as a silencer (Fig. $4 C$ ). In addition, because the $d l$-binding sites are intact, the VRE-MSE fusion gene directs weak expression along the entire ventral surface in response to peak levels of $d l$ (Jiang et al. 1993; Kirov et al. 1993).

A wild-type VRE-MSE fusion promoter containing an intact VRE directs an abnormal pattern of expression in embryos derived from $t^{4021} /+$ females (Fig. 4B). There is no ventral repression of the eve stripe 2 pattern; in
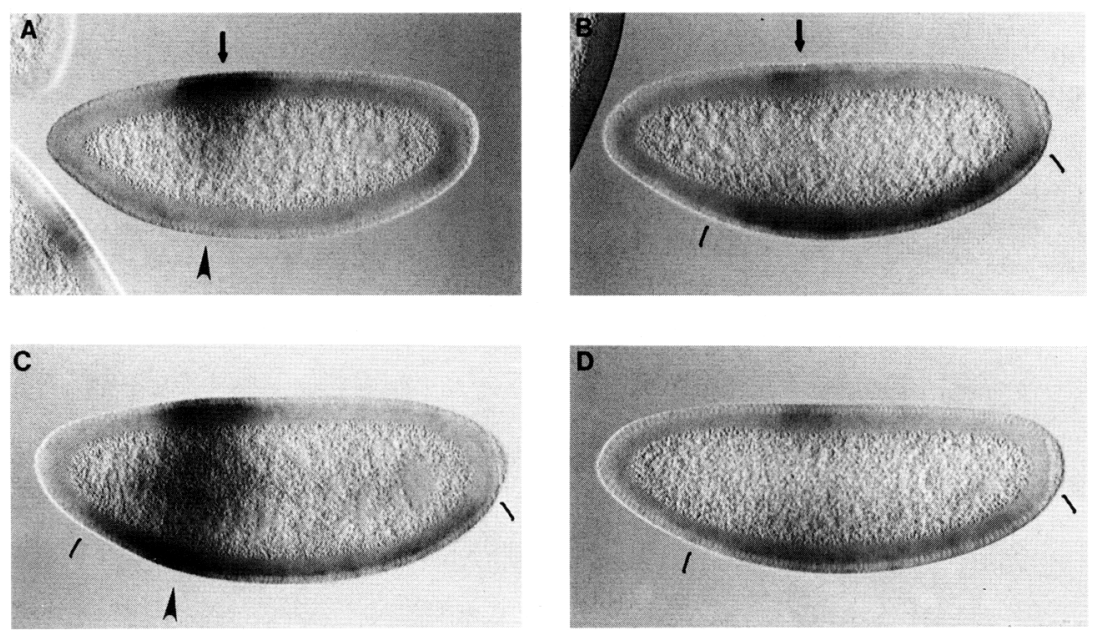

Figure 4. Constitutive activation of tor selectively disnupts $d l$-mediated repression. $(A, B)$ Expression of the $2 \times 180$ VRE-MSE fusion promoter in a wild-type $|A|$ and tor ${ }^{4021}$ embryo $(B)$. This promoter contains two tandem copies of a minimal zen silencer element (VRE) placed upstream of the eve stripe 2 enhancer (MSE). In wild-type embryos $(A)$, expression of the stripe 2 enhancer is restricted to dorsal regions and is repressed in ventral regions by the zen silencer (arrowhead). (B) There is no ventral repression in embryos derived from tor $^{4021} /+$ females. In addition, weak staining extends along the ventral surface where there are peak levels of $d l$ (indicated by brackets). The overall reduction in the levels of stripe 2 expression (see dorsal surface) is probably the result of the down-regulation of bcd by tor (see text $).(C, D)$ Expression of a mutagenized zen-eve fusion promoter, mVRE-MSE, in a wild-type embryo $(C)$ and in a mutant embryo derived from a tor ${ }^{4021} /+$ female $(D)$. This promoter contains one copy of the 180-bp VRE placed upstream of the eve MSE. Corepressor sites were eliminated in the VRE by site-directed mutagenesis. As a result, there is no ventral repression of the epression pattern in wild-type embryos $(C$; arrowhead). In addition, there is weak ventral activation by $d l$ (brackets). A similar expression pattern is observed in tor ${ }^{4021}$ mutants $(D)$, except that there is an overall reduction in the levels of stripe 2 expression. 
addition, there is weak staining along the ventral surface. This expression pattern is quite similar to that observed for the mutagenized VRE-MSE fusion promoter containing a defective VRE lacking corepressor binding sites (Fig. 4C). These results suggest that the constitutive activation of the tor RTK selectively blocks $d l$-corepressor interactions that are essential for $\mathrm{dl}$-mediated repression. However, tor does not interfere with the ability of $d l$ to bind the VRE and mediate weak activation. The reduced levels of staining directed by the eve MSE (see dorsal surfaces in Fig. 4A,B) might result from tor-bcd interactions. bcd is the primary activator of $e v e$ stripe 2, and previous studies have shown that tor attenuates $b c d$ activation at the anterior pole (Ronchi et al. 1993); it is reasonable to suppose that this $b c d$-tor interaction occurs in a more expanded domain in tor ${ }^{4021}$ mutants (see Discussion). A similar reduction in stripe 2 expression is observed when the mutagenized VRE-MSE fusion promoter lacking corepressor sites is expressed in a tor ${ }^{4021}$ background (Fig. 4D).

\section{tor-dl interactions are mediated by downstream signaling components}

Several intracellular kinases have been implicated in the tor signaling pathway, including the Drosophila homolog of the mammalian Raf serine/threonine kinase (D-raf; for review, see Perrimon 1993). To determine whether this intracellular pathway is also important for mediating the tor- $d l$ interaction, particularly the masking of $d l$-mediated transcriptional repression, we examined the expression of $d l$ target genes in embryos derived from eggs lacking $D$-raf ${ }^{+}$products. Because $D$-raf is an essential gene that is active throughout the Drosophila life cycle, germ-line mosaics were induced in $D-$ raf $^{+} / D$ raf $^{-}$heterozygotes. For this purpose, a heterozygous strain was employed that carries the yeast flip recombinase binding site (FRT) near the base of the chromosome $\mathrm{arm}$. The strain also contains the flip recombinase under the control of the heat-inducible $h s p 70$ promoter and a dominant female sterile mutation /Chou and Perrimon
1992; Melnick et al. 1993; Ronchi et al. 1993). When larvae are heat-shocked there is efficient recombination of the FRT-marked chromosomes, so that large $D$-raf ${ }^{-}$ patches of tissue are generated in the developing germ line. Nearly all of the eggs laid by these females lack $D$-raf $f^{+}$products, as judged by the loss of $t 11$ expression at the posterior pole (data not shown). Embryos derived from these females display an abnormal zen pattern, whereby expression is lost at the poles (Fig. 5B cf. B with A). This pattern is indistinguishable from that observed in embryos derived from tor $^{-}$females (Fig. 1B), suggesting that the ability of tor to mask $d l$ repression is mediated by the intracellular $D$-raf kinase.

Previous studies have shown that the repression of sna from the anterior and posterior poles is also controlled by tor. In tor $^{-}$mutants, sna expression is sustained at the posterior pole (Casanova 1991), whereas in wild-type embryos, this expression is extinguished during cellularization (Fig. 5C). This posterior repression probably is mediated by the tor target gene $h k b$ (M. Leptin, pers. comm.). Embryos derived from $D$-raf- eggs show a persistence of sna expression at the posterior pole (Fig. 5D), identical to the situation observed in tor ${ }^{-}$mutants. This observation suggests that intracellular signaling components downstream of the tor RTK and ras mediate sna repression at the posterior pole.

Recent studies on the sevenless (sev) RTK have identified a MAP kinase, rolled, that is essential for signal transduction in this pathway (Brunner et al. 1994; Biggs et al. 1994). A dominant, hyperactivated form of the rolled kinase, Sevenmaker (Sem), results in the formation of supernumerary R7 photoreceptor cells, similar to the situation observed for dominant gain-of-function mutations in the sev RTK (Brunner et al. 1994). Embryos derived from $\mathrm{Sem} /+$ females show segmentation defects that are consistent with an expanded pattern of $t 11$ expression (data not shown). However, disruptions in the segmentation pattern are erratic and generally not as severe as those observed in embryos carrying strong tor dominant mutations. To determine whether the rolled MAP kinase mediates aspects of the tor-dl interaction,
Figure 5. $D$-raf mediates the $d 1$-tor interaction. Embryos in $B$ and $D$ were obtained from $1(1) p h^{E A 75} /+$ females that were induced to deposit eggs completely lacking $D$-raf activity (see Materials and methods). $(A)$ zen expression pattern in a wild-type embryo. $(B)$ zen expression in an embryo lacking maternal $D$-raf products. Staining is lost from both poles, similar to the situation observed in tor ${ }^{-}$embryos (cf. Fig. 1B). (C) sna expression pattern in a wild-type embryo. Staining is lost from the poles (arrowheads) by this stage in development (late nuclear cycle 14). (D) sna expression pattern in embryos derived from $D$-raf ${ }^{-}$eggs. There is no repression from the poles, similar to the situation observed in tor $^{-}$.
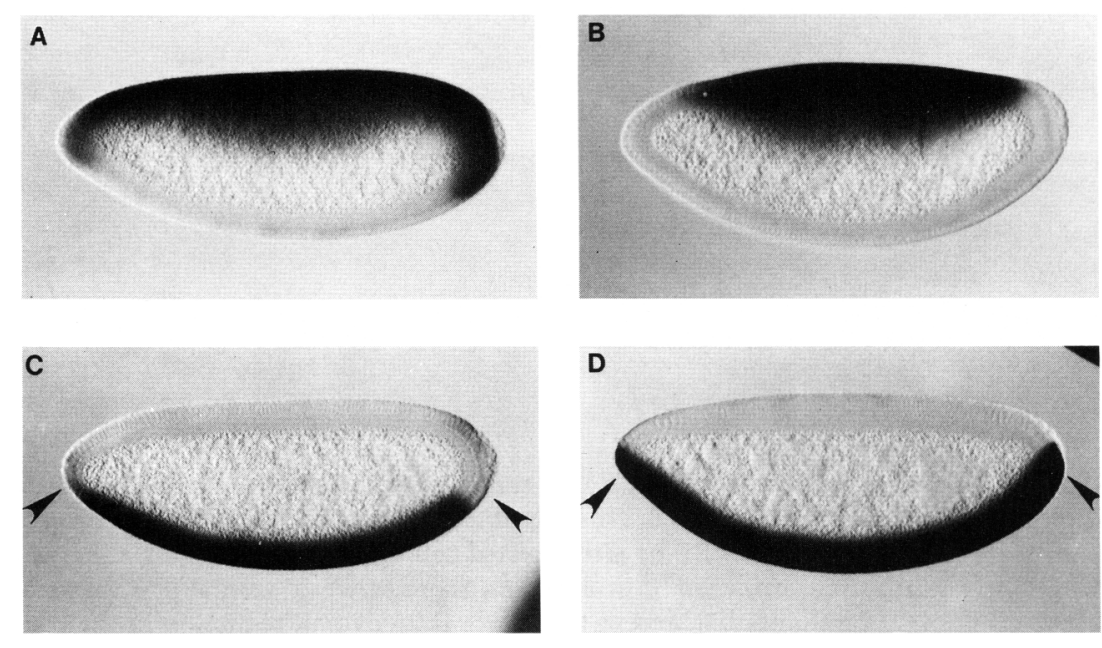
the zen expression pattern was examined in embryos derived from Sem/ + females (Fig. 6A). The zen pattern shows expanded limits of expression, such that staining extends into ventrolateral regions of the embryo where the gene is normally off (i.e., cf. Fig. 6A with Fig. 5A). This observation suggests that hyperactivation of the rolled MAP kinase causes $d l$ to be a less effective repressor.

Further evidence that rolled attenuates $d l$ repression was obtained by examining the expression of the zen VRE-eve MSE fusion promoter in embryos derived from $\mathrm{Sem} /+$ females (Fig. 6B). The MSE drives strong expression in both dorsal and ventral regions, with only a slight reduction in ventral regions. In wild-type embryos, staining is restricted to dorsal regions because the VRE silences ventral expression (cf. Fig. 6B with Fig. 4A). This result suggests that the hyperactivated MAP kinase impairs, but does not abolish, $d l$-mediated repression from the zen VRE. However, the effect is not as dramatic as that observed in tor $^{D}$ mutants (i.e., Fig. $4 \mathrm{~B}$ ), suggesting that the rolled MAP kinase might play a relatively dedicated role in transmitting the sev signal (see Discussion).

\section{Discussion}

dl functions as both a sequence-specific transcriptional activator and repressor (for review, see Ip and Levine 1992). Here, we present evidence that the tor RTK signaling pathway fine-tunes $d l$ activity by selectively blocking its ability to repress target genes such as zen. This block in repression helps "dorsalize" the embryonic poles and delimit the anteroposterior boundaries of the presumptive mesoderm. Evidence is presented that the $D$-raf serine-threonine kinase transduces the signal generated by the activation of the tor receptor. The newly identified rolled MAP kinase appears to function downstream of $D$-raf to suppress $d l$ repression, but it is possible that additional, as yet unidentified, intracellular signaling components also participate in this process. These findings, together with previous studies on the Toll signaling pathway (for review, see Govind and Steward 1991; Wasserman 1993), suggest that the detailed activities of a single transcription factor can be modu- lated by multiple pathways. Thus, the $d l$ morphogen is subject to exquisite regulation: Its activity is influenced by divergent signaling pathways and the context of its target promoters (Jiang et al. 1992; Pan and Courey 1992).

\section{Convergence of multiple signaling pathways}

The most important finding of this study is that the tor RTK pathway exerts a relatively specific effect on $d l$ mediated repression and that multiple signaling pathways can act on a single transcription factor, as summarized in Figure 7. However, it should be emphasized that the tor pathway might not lead to the direct modification of $d l$, but, instead, could function by modulating one or more $d l$ corepressors (see Huang et al. 1993; Jiang et al. 1993; Kirov et al. 1993, 1994). The Toll pathway is activated in both ventral regions and the poles by the spätzle ligand (Anderson et al. 1992; Morisato and Anderson 1994). This leads to the dissociation of the inactive dl-cact complex so that $d l$ can enter the nucleus and function as both an activator of twi and sna and a repressor of zen and $d p p$ (Govind and Steward 1991; Ip and Levine 1992; Wasserman 1993). Both the Toll and tor pathways are active at the poles. As a result, $d l$ activates twi and sna but fails to repress zen and $d p p$, so all of these genes are expressed at the poles.

\section{Role of tor in dorsoventral patterning}

Previous studies on the tor pathway have focused primarily on segmentation. The follicular epithelium surrounding the developing oocyte is thought to secrete a tor ligand, possibly encoded by tsl, only at the polar regions of the egg chamber (Stevens et al. 1990; SavantBhonsale and Montell 1993; Martin et al. 1994). This leads to the selective activation of the tor RTK at the poles and localized expression of two target genes, $t 11$ and $h k b$ (Brönner and Jäckle 1991; Liaw and Lengyel 1993). Embryos derived from tor ${ }^{-}$females lack the posterior domain of $t l l$ expression and display an abnormal pattern of expression at the anterior pole (Pignoni et al. 1990). Most of the segmentation defects observed in tor mutants appear to result from altered expression of $t 11$ [Ste-
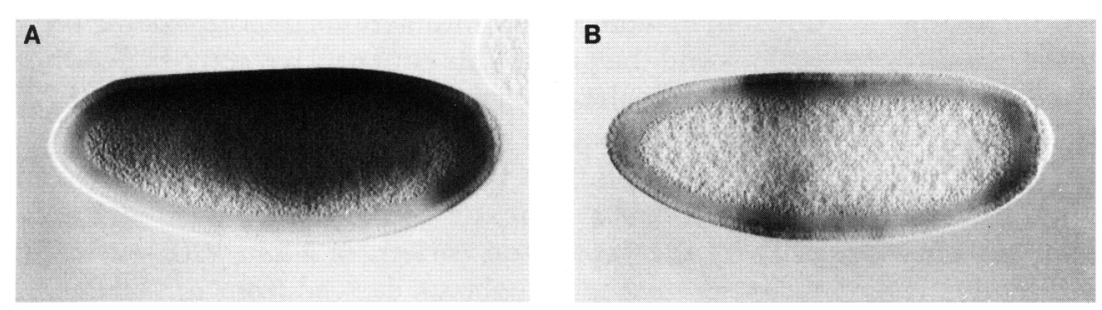

Figure 6. Hyperactivation of the rolled MAP kinase disrupts $d l$-mediated repression. Embryos were collected from Sem $/+$ females. (A) zen expression pattern. There is a slight ventral derepression of the pattern so that staining extends into ventrolateral regions where the gene is normally off (cf. with Figs. 1A, 2A, and 5A). However, this derepression is not as severe as that observed in the tor ${ }^{4021}$ background (cf. Fig. 2B). (B) Expression of the $2 \times 180$ VRE-MSE fusion promoter. Stripe 2 is expressed at nearly uniform levels in both dorsal and ventral regions, indicating that $d l$ fails to mediate efficient repression in this mutant. In wild-type embryos, stripe 2 expression is restricted to the dorsal surface and there is complete ventral repression (see Fig. 4A). However, unlike the situation observed in tor ${ }^{4021}$ mutants, there is no staining detected along the ventral surface nor attenuation of the stripe 2 pattern /see Fig. 4B). 
Figure 7. Coordinate regulation of $d l$ by the tor and Toll signaling pathways. The Toll receptor is selectively activated in ventral regions, and the poles, by the spätzle ligand. This induces two cytoplasmic proteins, tube and pelle, to modify the dl-cact complex so that $d l$ is released from the cytoplasm and enters nuclei. Once in the nucleus, $d l$ acts as both activator of zygotic target genes such as twi and sna and as repressor of zen and dpp. In middle body regions, the tor receptor tyrosine kinase is expressed but not activated because of the lack of $t s l$ ligand. At the poles, the ligand activates tor and induces an intracellular signaling pathway that includes the products of $1(1) \mathrm{ph}(D$-raf $)$ and rolled (MAP kinase). This leads to a specific impairment in the ability of $d l$ to repress targets like zen and $d p p$ but has only slight effects on its ability to activate genes like twi and sna. The mechanism might involve modification of the $\mathrm{dl}$ protein itself, e.g., by phosphorylation, or of other components interacting with $d l$ in the repression of target genes. For example, the tor pathway might modify one or more $d l$ corepressors and thereby block the formation of a $d l$ repression complex on the zen promoter.
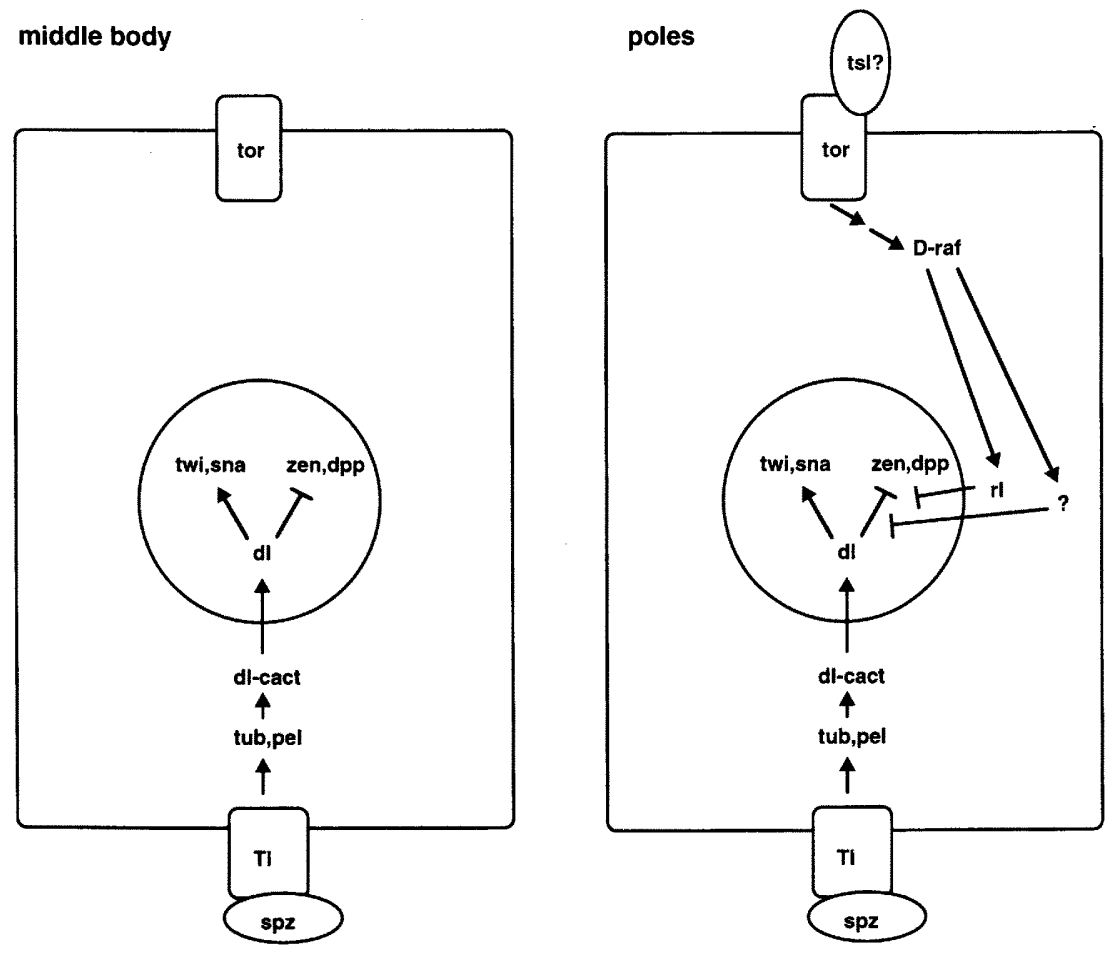

ingrímsson et al. 1991). For example, in strong tor ${ }^{D} \mathrm{mu}-$ tants there is ectopic expression of $t l l$, which in some cases encompasses nearly the entire length of the embryo (i.e., Fig. 2D). There is a tight correlation between the extent to which tll is ectopically expressed and the suppression of middle body segments. In severe cases there is a virtual loss of all segments, whereas slight expansion of the tll pattern results in the loss of just one or two abdominal segments (Warrior and Levine 1990). Most of the segmentation defects observed in $\operatorname{tor}^{D} \mathrm{mu}-$ tants can be suppressed by removing $t 1 l^{+}$gene activity (Strecker et al. 1991).

There is evidence that tor also plays an important role in dorsoventral patterning. Some of the head defects observed in tor ${ }^{-}$mutants could result from the loss of zen at the anterior pole, because $z e n^{+}$gene activity is required for the differentiation of head structures and is also important for head involution (Wakimoto et al. 1984). In addition, there are a number of dorsoventral patterning defects observed in tor $^{D}$ mutants, including disruptions in ventral furrow formation, mesoderm differentiation, and germ-band elongation (J. Rusch and $M$. Levine, unpubl.). Finally, tor $^{+}$gene activity is required for repressing sna at the posterior pole; in tor ${ }^{-}$mutants, sna expression is sustained in these regions and there is a corresponding expansion of the ventral furrow and embryonic mesoderm (Casanova 1991). Consequently, it would appear that tor is important for defining the anteroposterior boundaries of the ventral furrow.

Mechanism of tor-dl interaction

$d l$ functions as a repressor that restricts the expression of tissue determinants such as zen and $d p p$ to dorsal regions where they are important for initiating the differentiation of the dorsal ectoderm (Rushlow et al. 1987; St Johnston and Gelbart 1987). Repression involves local protein-protein interactions between $d l$ and neighboring corepressors bound to zen and $d p p$ silencer elements (Jiang et al. 1992, 1993; Pan and Courey 1992; Huang et al. 1993; Kirov et al. 1993). These interactions convert the weak $d l$ activator into a long-range repressor that can silence the ventral expression of various heterologous promoters, including hunchback, Krüppel, and eve (Doyle et al. 1989; Ip et al. 1991). It would appear that the tor pathway selectively blocks $d l$-corepressor interactions, thereby permitting zen and $d p p$ expression at the poles where there are high levels of $d l$ protein. In principle, dl-corepressor interactions could be disrupted by either modification of $d l$ or the corepressor. Constitutive activation of the tor RTK results in ubiquitous expression of zen and $d p p$, presumbably because of a breakdown in $d l$-corepressor interactions in both middle body regions and the poles. This constitutive activity does not significantly alter the expression of target genes that are activated by $d l$, such as twi and sna.

The uncoupling in the ability of $d l$ to function as an activator versus repressor is illustrated most dramatically by the expression pattern of a zen VRE-eve MSE fusion promoter in embryos derived from $t^{4021} /+\mathrm{fe}-$ males (Fig. 4). The VRE not only fails to silence the ventral expression of eve stripe 2 but actually mediates weak activation in response to peak levels of $d l$ present along the ventral surface. The block in $d l$-corepressor interactions does not result from a general failure of $d l$ to con- 
tact cooperating factors in a target promoter element. For example, activation of the synthetic PE-Et promoter throughout the presumptive mesoderm in response to both high and intermediate levels of $d l$ depends on cooperative DNA-binding interactions between $d l$ and HLH proteins bound to neighboring E-box sequences (Jiang and Levine 1993). The dorsoventral limits of the PE-Et pattern are not altered in embryos derived from tor $^{4021} /+$ females, suggesting that $d l-$ HLH interactions are unaffected (Fig. 3; data not shown).

\section{RTK pathways and antirepression}

A number of intracellular signaling molecules mediate the tor RTK signal. These include corkscrew, a tyrosine phosphatase; Son of sevenless; ras1; D-raf; Dsor1, a homolog of MEK kinase (MAP kinase kinase); and most recently, rolled/Sem, a putative MAP kinase (for review, see Perrimon 1993; Brunner et al. 1994; Biggs et al. 1994). Several of these signaling components have been implicated in other RTK pathways, including the epidermal growth factor (EGF) and sev RTKs (Dickson et al. 1992). However, it is not clear whether all of these components are equally important for the different RTKs.

For example, Sem/ + heterozygotes display a severe eye phenotype, extra R7 cells, which is quite similar to that observed upon constitutive activation of the $\mathrm{sev}$ RTK or ras1 (Brunner et al. 1994). This observation suggests that the dominant Sem mutation represents a hyperactivated form of the rolled MAP kinase. However, the kinase might play a variable role in the EGF RTK pathway because $\mathrm{Sem} /+$ females lay normal eggs but display extra wing veins; both of these processes are regulated by the $E G F$ receptor /Clifford and Schüpbach 1992). The observation that the $t 11$ and zen expression patterns are only mildly disrupted in embryos derived from Sem/ + eggs suggests that the rolled kinase might also play a limited role in transducing the tor signal. Perhaps the efficient derepression of zen depends on the activation of additional, as yet unidentified, signaling components that function downstream of ras 1 and $D$-raf.

RTKs have been implicated in the activation of gene expression in a variety of systems, including the Caenorhabditis elegans vulva (Sternberg and Horvitz 1991) and the fly ovary (Shilo 1992), embryo (Sprenger et al. 1989; Casanova and Struhl 1989), and eye (Dickson et al. 1992) and numerous mammalian tissues including $\mathrm{T}$ cells (e.g., Margolis et al. 1992). It is conceivable that one or more of these processes depend on the same type of antirepression mechanism underlying the dl-tor interaction. tor does not regulate zen and $d p p$ expression by inducing a transcriptional activator. Instead, it masks the action of a transcriptional repressor. Previous studies suggest that zen is activated by one or more ubiquitously distributed factors, which interact with multiple elements distributed throughout the zen promoter (Doyle et al. 1989; Jiang et al. 1993). The distal VRE silences this activation whenever $d l$ and its corepressors are bound to the VRE. dl-mediated repression is relieved at the poles by the tor RTK pathway. Perhaps $t l l$ expression is restricted to the poles by the masking of a ubiquitous repressor, and it is conceivable that the activation of R7specific genes also involves antirepression by the sev RTK (Lai and Rubin 1992).

\section{Materials and methods}

Fly stocks

Fly stocks used in this study were $y w^{67 c 23}, \mathrm{dl}^{15}$ (Steward and Nüsslein-Volhard 1986), tor ${ }^{P M 51}$ (Schüpbach and Wieschaus 1986), and tor ${ }^{4021}$ (Klingler et al. 1988). The tor ${ }^{P M 51}$; $d l^{15}$ double mutant was obtained by recombination. Germ-line $1(1) p h^{E A 75}$ (D-raf) mutant females were obtained by inducing site-specific mitotic recombination using the FLP-DFS technique /Chou and Perrimon 1992). X chromosomes have been constructed containing an FRT sequence near the centromere and either the dominant female sterile $O v O^{D 1}$ or the $1(1) p h^{E A 75}$ mutation. Males carrying the FRT $o v o^{D 1}$ chromosome and the flp recombinase under heat shock control on the second chromosome (F38) were crossed to females carrying the FRT 1(1)ph ${ }^{E A 75}$ chromosome and F38. These parental strains were kindly provided by Norbert Perrimon (Harvard Medical School; Boston, MA) and Claude Desplan (Rockefeller University, NY). The progeny of this cross were heat-shocked during the first-instar larval stage for $2 \mathrm{hr}$ at $37^{\circ} \mathrm{C}$ and allowed to develop at $25^{\circ} \mathrm{C}$. The fertile female progeny laid eggs that developed with a strong terminal phenotype as shown by eve antibody stainings (data not shown). Sem mutant females were obtained by outcrossing males from a $1(1) \mathrm{ph}^{\mathrm{HM} 7} ; \mathrm{Sem} /+$ stock to $\mathrm{yw}$ females and selecting for the Sem phenotype (rough eyes and extra wing veins) among the female offspring. The Sem strain was kindly provided by Ernst Hafen (Zoologisches Institut Universität Zürich, Switzerland).

Transgenic lines containing various $l a c Z$ fusion genes have been described previously: 1.6-kb sna-lacZ (Ip et al. 1992b); PE-Et (Jiang and Levine 1993); $2 \times 180$ VRE-MSE (Jiang et al. 1993). To examine the expression of the various $l a c Z$ fusion genes in mutant backgrounds, males carrying a given fusion gene were crossed with females of the appropriate genotype. For the wt background, yw females were used. All embryo collections were done at $25^{\circ} \mathrm{C}$.

\section{Whole mount in situ hybridization}

Embryonic expression patterns were visualized by in situ hybridization using antisense RNA probes labeled with digoxygenin-UTP and antidigoxygenin antibodies conjugated to alkaline phosphatase (Tautz and Pfeifle 1989; Jiang et al. 1991; Kosman et al. 1991). The stained embryos were photographed using Nomarski optics.

\section{Acknowledgments}

We thank Stephen Small, Rick Firtel, Jim Posakony, and Ethan Bier for helpful suggestions and encouragement. We also thank Ernst Hafen and Claude Desplan for providing fly stocks. This study was funded by a grant from the National Institutes of Health (GM 46638).

The publication costs of this article were defrayed in part by payment of page charges. This article must therefore be hereby marked "advertisement" in accordance with 18 USC section 1734 solely to indicate this fact. 


\section{References}

Alberga, A., J.-L. Boulay, E. Kempe, C. Dennefeld, and M. Haenlin. 1991. The snail gene required for mesoderm formation in Drosophila is expressed dynamically in derivatives of all three germ layers. Development 111: 983-992.

Anderson, K.V., D.S. Schneider, D. Morisato, Y. Jin, and E.L. Ferguson. 1992. Extracellular morphogens in Drosophila embryonic dorsal-ventral patterning. Cold Spring Harbor Symp. Quant. Biol. 57: 409-417.

Biggs W.H. III, K.H. Zavitz, B. Dickson, A. van der Straten, D. Brunner, E. Hafen, and S.L. Zipursky. 1994. The Drosophila rolled locus encodes a MAP kinase required in the sevenless signal transduction pathway. EMBO I. 13: 1628-1635.

Boulay, J.-L., C. Dennefeld, and A. Alberga. 1987. The Drosophila developmental gene snail encodes a protein with nucleic acid binding fingers. Nature 330: 395-398.

Brönner, G. and H. Jäckle. 1991. Control and function of terminal gap gene activity in the posterior pole region of the Drosophila embryo. Mech. Dev. 35: 205-211.

Brunner, D., N. Oellers, J. Szabad, and E. Hafen. 1994. A gain of function mutation in Drosophila MAP kinase constitutively activates multiple receptor tyrosine kinase signalling pathways. Cell 76: 875-888.

Casanova, J. 1991. Interaction between torso and dorsal, two elements of different transduction pathways in the Drosophila embryo. Mech. Dev. 36: 41-45.

Casanova, J. and G. Struhl. 1989. Localized surface activity of torso, a receptor tyrosine kinase, specifies terminal body pattern in Drosophila. Genes \& Dev. 3: 2025-2038.

Chou, T.-B. and N. Perrimon. 1992. Use of a yeast site-specific recombinase to produce female germline chimeras in Drosophila. Genetics 131: 643-653.

Clifford, R. and T. Schüpbach. 1992. The torpedo (DER) receptor tyrosine kinase is required at multiple times during Drosophila embryogenesis. Development 115: 853-872.

Dickson, B., F. Sprenger, D. Morrison, and E. Hafen. 1992. Raf functions downstream of ras1 in the sevenless signal transduction pathway. Nature 360: 600-603.

Doyle, H.J., K. Harding, T. Hoey, and M. Levine. 1986. Transcripts encoded by a homeo box gene are restricted to dorsal tissues of Drosophila embryos. Nature 323: 76-79.

Doyle, H.J., R. Kraut, and M. Levine. 1989. Spatial regulation of zerknüllt: A dorsal-ventral patterning gene in Drosophila. Genes \& Dev. 3: 1518-1533.

Geisler, R., A. Bergmann, Y. Hiromi, and C. Nüsslein-Volhard. 1992. cactus, a gene involved in dorsoventral pattern formation of Drosophila, is related to the IкB gene family of vertebrates. Cell 71: 613-621.

González-Crespo, S. and M. Levine. 1993. Interactions between dorsal and helix-loop-helix proteins initiate the differentiation of the embryonic mesoderm and neuroectoderm in Drosophila. Genes \& Dev. 7: 1703-1713.

Govind, S. and R. Steward. 1991. Dorsoventral pattern formation in Drosophila: Signal transduction and nuclear targeting. Trends Genet. 7: 119-125.

Huang, J.-D., D.H. Schwyter, J.M. Shirokawa, and A.J. Courey. 1993. The interplay between multiple enhancer and silencer elements defines the pattern of decapentaplegic expression. Genes \& Dev. 7: 694-704.

Ip, Y.T. and M. Levine. 1992. The role of the dorsal morphogen gradient in Drosophila embryogenesis. Sem. Dev. Biol. 3: $15-23$.

Ip, Y.T., R. Kraut, M. Levine, and C. Rushlow. 1991. The dorsal morphogen is a sequence-specific DNA-binding protein that interacts with a long-range repression element in Droso- phila. Cell 64: 439-446.

Ip, Y.T., R.E. Park, D. Kosman, E. Bier, and M. Levine. 1992a. The dorsal gradient morphogen regulates stripes of rhomboid expression in the presumptive neuroectoderm of the Drosophila embryo. Genes \& Dev. 6: 1728-1739.

Ip, Y.T., R.E. Park, D. Kosman, K. Yazdanbakhsh, and M. Levine. 1992b. dorsal-twist interactions establish snail expression in the presumptive mesoderm of the Drosophila embryo. Genes \& Dev. 6: 1518-1530.

Jiang, J. and M. Levine. 1993. Binding affinities and cooperative interactions with bHLH activators delimit threshold responses to the dorsal gradient morphogen. Cell 72: 741-752.

Jiang, J., D. Kosman, Y.T. Ip, and M. Levine. 1991. The dorsal morphogen gradient regulates the mesoderm determinant twist in early Drosophila embryos. Genes \& Dev. 5: 18811891.

Jiang, J., C.A. Rushlow, Q. Zhou, S. Small, and M. Levine. 1992. Individual dorsal morphogen binding sites mediate activation and repression in the Drosophila embryo. EMBO J. 11: 3147-3154.

Jiang, J., H. Cai, Q. Zhou, and M. Levine. 1993. Conversion of a dorsal-dependent silencer into an enhancer: Evidence for dorsal corepressors. EMBO I. 12: 3201-3209.

Kidd, S. 1992. Characterization of the Drosophila cactus locus and analysis of interactions between cactus and dorsal proteins. Cell 71: 623-635.

Kirov, N., L. Zhelnin, J. Shah, and C. Rushlow. 1993. Conversion of a silencer into an enhancer: Evidence for a co-repressor in dorsal-mediated repression in Drosophila. EMBO \%. 12: 3193-3199.

Kirov, N., S. Childs, M. O'Connor, and C. Rushlow. 1994. The Drosophila dorsal morphogen represses the tolloid gene by interacting with a silencer element. Mol. Cell. Biol. 14: 713722.

Klingler, M., M. Erdélyi, J. Szabad, and C. Nüsslein-Volhard. 1988. Function of torso in determining the terminal anlagen of the Drosophila embryo. Nature 335: 275-277.

Kosman, D., Y.T. Ip, M. Levine, and K. Arora. 1991. Establishment of the mesoderm-neuroectoderm boundary in the Drosophila embryo. Science 254: 118-122.

Lai, Z.C. and G.M. Rubin. 1992. Negative control of photoreceptor development in Drosophila by the product of the yan gene, an ETS domain protein. Cell 70: 609-620.

Leptin, M. 1991. twist and snail as positive and negative regulators during Drosophila mesoderm development. Genes \& Dev. 5: 1568-1576.

Liaw, G.-J. and J.A. Lengyel. 1993. Control of tailless expression by bicoid, dorsal and synergistically interacting terminal system regulatory elements. Mech. Dev. 40: 47-61.

Liou, H.C. and D. Baltimore. 1993. Regulation of the NF kappa B/rel transcription factor and I kappa B inhibitor system. Curr. Opin. Cell Biol. 5: 477-487.

Margolis, B., P. Hu, S. Katzav, W. Li, J.M. Oliver, A. Ullrich, A. Weiss, and J. Schlessinger. 1992. Tyrosine phosphorylation of vav proto-oncogene product containing $\mathrm{SH} 2$ domain and transcription factor motifs. Nature 356: 71-74.

Martin, J.-R., A. Raibaud, and R. Ollo. 1994. Terminal pattern elements in Drosophila embryo induced by the torso-like protein. Nature 367: 741-745.

Melnick, M.B., L.A. Perkins, M. Lee, L. Ambrosio, and N. Perrimon. 1993. Developmental and molecular characterization of mutations in the Drosophila-raf serine/threonine protein kinase. Development 118: 127-138.

Morisato, D. and K.V. Anderson. 1994. The spätzle gene encodes a component of the extracellular signaling pathway establishing the dorsal-ventral pattern of the Drosophila em- 
bryo. Cell 76: 677-688.

Pan, D. and A.J. Courey. 1992. The same dorsal binding sites mediate both activation and repression in a context-dependent manner. $E M B O$ I. 11: 1837-1842.

Pan, D., J.-D. Huang, and A.J. Courey. 1991. Functional analysis of the Drosophila twist promoter reveals a dorsal-binding ventral activator regions. Genes \& Dev. 5: 1892-1901.

Perrimon, N. 1993. The torso receptor protein-tyrosine kinase signaling pathway: An endless story. Cell 74: 219-222.

Pignoni, F., R.M. Baldarelli, E. Steingrímsson, R.J. Diaz, A. Patapoutian, J.R. Merriam, and J.A. Lengyel. 1990. The Drosophila gene tailless is expressed at the embryonic termini and is a member of the steroid receptor superfamily. Cell 62: 151-163.

Pignoni, F., E. Steingrímsson, and J.A. Lengyel. 1992. bicoid and the terminal system activate tailless expression in the early Drosophila embryo. Development 115: 239-251.

Ronchi, E., J. Treisman, N. Dostatni, G. Struhl, and C. Desplan. 1993. Down-regulation of the Drosophila morphogen bicoid by the torso receptor-mediated signal transduction cascade. Cell 74: 347-355.

Roth, S., D. Stein, and C. Nüsslein-Volhard. 1989. A gradient of nuclear localization of the dorsal protein determines dorsoventral pattern in the Drosophila embryo. Cell 59: 11891202.

Rushlow, C., M. Frasch, H. Doyle, and M. Levine. 1987. Maternal regulation of zerknüllt: A homeobox gene controlling differentiation of dorsal tissues in Drosophila. Nature 330: 583-586.

Rushlow, C.A., K. Han, J.L. Manley, and M. Levine. 1989. The graded distribution of the dorsal morphogen is initiated by selective nuclear transport in Drosophila. Cell 59: 11651177.

Savant-Bhonsale, S. and D.J. Montell. 1993. torso-like encodes the localized determinant of Drosophila terminal pattern formation. Genes \& Dev. 7: 2548-2555.

Schüpbach, T. and E. Wieschaus. 1986. Maternal-effect mutations altering the anterior-posterior pattern of the Drosophila embryo. Wilhelm Roux's Arch. Dev. Biol. 195: 302317.

Shilo, B.-Z. 1992. Roles of receptor tyrosine kinases in Drosophila development. FASEB J. 6: 2915-2922.

Simpson, P. 1983. Maternal-zygotic gene interactions during formation of the dorsoventral pattern in Drosophila embryos. Genetics 105: 615-632.

Sprenger, F., L.M. Stevens, and C. Nüsslein-Volhard. 1989. The Drosophila gene torso encodes a putative receptor tyrosine kinase. Nature 338: 478-483.

Steingrímsson, E., F. Pignoni, G.-J. Liaw, and J.A. Lengyel. 1991. Dual role of the Drosophila pattern gene tailless in embryonic termini. Science 254: 418-421.

Sternberg, P.W. and H.R. Horvitz. 1991. Signal transduction during C. elegans vulval induction. Trends Genet. 7: 366371.

Stevens, L., H.G. Frohnhöfer, M. Klingler, and C. Nüsslein-Volhard. 1990. Localized requirement for torso-like expression in follicle cells for development of terminal anlagen of the Drosophila embryo. Nature 346: 660-663.

Steward, R. 1987. Dorsal, an embryonic polarity gene in Drosophila, is homologous to the vertebrate proto-oncogene, c-rel. Science 238: 692-694.

- 1989. Relocalization of the dorsal protein from the cytoplasm to the nucleus correlates with its function. Cell 59: 1179-1188.

Steward, R. and C. Nüsslein-Volhard. 1986. The genetics of the dorsal-Bicaudal-D region of Drosophila melanogaster. Ge- netics 113: 665-678.

St Johnston, D. and W.M. Gelbart. 1987. decapentaplegic transcripts are localized along the dorsal-ventral axis of the Drosophila embryo. EMBO J. 6: 2785-2791.

St Johnston, D. and C. Nüsslein-Volhard. 1992. The origin of pattern and polarity in the Drosophila embryo. Cell 68: 201219.

Strecker, T., M.L.R. Yip, and H.D. Lipshitz. 1991. Zygotic genes that mediate torso receptor tyrosine kinase functions in the Drosophila melanogaster embryo. Proc. Natl. Acad. Sci. 88: 5824-5828.

Tautz, D. and C. Pfeifle. 1989. A non-radioactive in situ hybridization method for the localization of specific RNAs in Drosophila reveals a translational control of the segmentation gene hunchback. Chromosoma 98: 81-85.

Thisse, B., C. Stoetzel, M.E. Messal, and F. Perrin-Schmitt. 1987. Genes of the Drosophila maternal dorsal group control the specific expression of the zygotic gene twist in presumptive mesodermal cells. Genes \& Dev. 1: 709-715.

Thisse, C., F. Perrin-Schmitt, C. Stoetzel, and B. Thisse. 1991. Sequence-specific transactivation of the Drosophila twist gene by the dorsal gene product. Cell 65: 1191-1201.

Wakimoto, B.T., F.R. Turner, and T.C. Kaufman. 1984. Defects in embryogenesis in mutants associated with the Antennapedia gene complex of Drosophila melanogaster. Dev. Biol. 102: $147-172$.

Warrior, R. and M. Levine. 1990. Dose-dependent regulation of pair-rule stripes by gap proteins and the initiation of segment polarity. Development 110: 759-767.

Wasserman, S.A. 1993. A conserved signal transduction pathway regulating the activity of the rel-like proteins dorsal and NFкB. Mol. Biol. Cell 4: 767-771. 


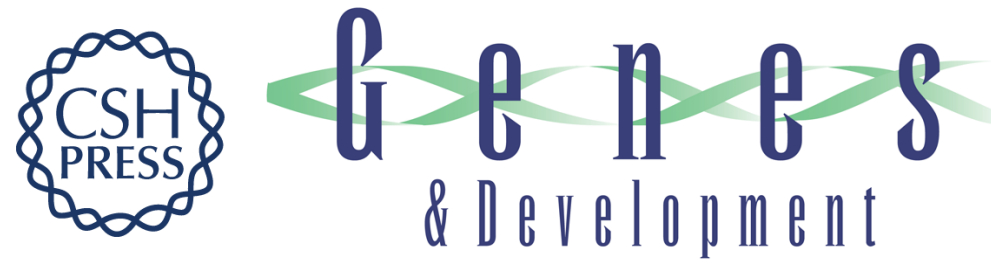

\section{Regulation of the dorsal morphogen by the Toll and torso signaling pathways: a receptor tyrosine kinase selectively masks transcriptional repression.}

J Rusch and M Levine

Genes Dev. 1994, 8:

Access the most recent version at doi:10.1101/gad.8.11.1247

References This article cites 67 articles, 26 of which can be accessed free at:

http://genesdev.cshlp.org/content/8/11/1247.full.html\#ref-list-1

License

Email Alerting Service

Receive free email alerts when new articles cite this article - sign up in the box at the top right corner of the article or click here.

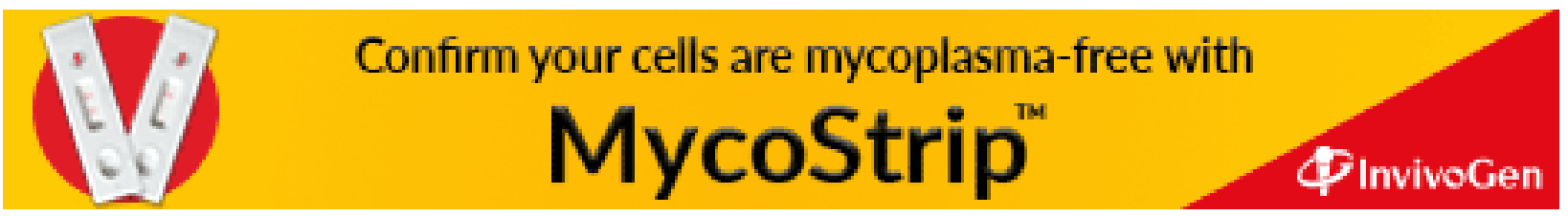

